\title{
WHAT CAN BE BETTER THAN THE BEST?
}

\author{
KLÁRA ŠEĎOVÁ
}

\begin{abstract}
A Book Review
Lefstein, A., Snell, J. (2014). Better than "best practice": Developing teaching and learning through dialogue. London: Routledge.
\end{abstract}

The book by Adam Lefstein and Julia Snell explores a wide array of dialogic pedagogy. ${ }^{1}$ The authors show what really happens in classes where teachers apply dialogic teaching methods, using data from an action research project implemented in 2008-2009 at a London school. The researchers prepared a Professional Development Programme ${ }^{2}$ for teachers. During its implementation, they observed the participating teachers in their literacy lessons. They collected a solid corpus of data, including video recordings of the lessons, recordings of the workshops for the teachers with discussions, and individual in-depth interviews with the teachers.

Only a very small part of this extensive material was used in the book. Each of the core chapters are organized with respect to the transcript of a selected teaching episode, lasting a few minutes. However, because of the detailed approach that the authors take to the data, an analysis of more material is difficult to imagine.The book contains very erudite analytical insights that academic experts will read with pleasure, but the book is primarily designed to help in-service teachers develop their own teaching style.

1 Dialogic pedagogy in the book is seen as an umbrella term for a range of different approaches that share an emphasis on the use of dialogue as form of interaction in teaching and learning. Dialogic teaching is defined, for example, by Alexander (2006, p. 37) as a way of using the "power of talk to engage children, stimulate and extend their thinking, and advance their learning and understanding."

2 In order to familiarize teachers with dialogic teaching methods and help them implement these methods in their lessons. 
The title of the book is provocative: what could be better than the best practice? According to Lefstein and Snell, the concept of best practice is shaped by the idea of gifted teachers developing procedures that will be adopted by others. This type of imitation, however, faces a well-known problem: a method that works in one context and for one teacher may not work in another context or for another teacher. Teachers should have a certain repertoire of methods, and it is also necessary for them to develop their abilities to observe, interpret, and discuss pedagogical phenomena. This is the main mission of the book. The selected individual episodes around which each chapter is built serve as springboards for discussion about various phenomena in dialogic teaching. Questions are raised gradually, concerning the circumstances in which the normal IRE structure ${ }^{3}$ may transform into an authentic dialogue (Chapter 4), how to deal with student challenges (Chapter 5), the potential results of importing popular culture into the classroom (Chapter 6), the methods of controlling a debate among students (Chapter 7), and how student capabilities and socially constructed identities determine their participation in the dialogues (Chapter 8).

The reader is not informed about why certain episodes were selected or about the way the key themes were identified, which can be seen as a weak point of the book. We do not know whether the episodes were selected before the topics for analysis emerged or if the topics were selected first and then the episodes were chosen to illustrate them.

Although each chapter has a key theme, the analysis is never purely focused on this topic. The authors pay attention to many aspects of each specific situation, often arranging it as a kind of dilemma. In accordance with the concept of the book, ideal examples of dialogic teaching are not revealed. Instead, real-life situations that teachers find themselves in are mapped, and the logic of the processes the teachers select and the effects of these processes are reviewed. A number of different and often contradictory effects always follow from even a single decision by a teacher, some intentional and conscious, some unintentional and unconscious. Chapter 6, for example, presents an episode in which a teacher wants the students to evaluate the written works of their classmates. To increase their interest and motivation, the teacher arranges the situation as an assessment similar to the TV talent

IRE structure (see Mehan, 1979) consists of three components: the initiation of the teacher - the response of the student - the evaluation of the teacher. IRE is seen as the basic interaction mechanism of teacher communication. Rigid adherence to this structure is criticized within dialogic pedagogy as restrictive to student participation and thinking space. 
contest "The X Factor". This plan is successfully executed: the students are actually very active in their communication, the teacher recedes into the background, and the whole sequence is very different from the traditional IRE structure. At the same time, the implementation of the genre evokes exaggerated criticism from the students as they imitate the behavior of the jurors in the TV contest. The decision, which is productive in one respect (increased participation), thus proves to be problematic in another respect (providing realistic and formative feedback).

The authors postulate that dialogic pedagogy views a dialogue as a problem rather than a solution. This view corresponds with the emphasis given to the dilemmas and tensions that the teachers experience. This belief is also reflected by the very organization of the chapters. Each chapter begins with a narrative description of the presented episode and background information concerning the lesson, the students, and the teacher. This is followed by a transcript of the episode, after which the readers are invited to watch a videotape of the episode on the project website, where they are given a few questions to focus on while watching the video. In the next part, the authors subject the episode to a detailed analysis and then create more questions for the reader. The end of each chapter contains additional comments on the opinions concerning the episode provided by selected specialists on dialogic teaching - mostly respected academics, but also practitioners from institutions concerned with teacher development or from schools. ${ }^{4}$

The book is presented in a very original form, allowing for many different voices: the authors, teachers who participated in the research, contributing commentators, and readers themselves. ${ }^{5}$ It is a very appropriate form for the topic - it could described as a dialogically conceived book about dialogic teaching. This is valuable, because there is currently a gap between the theory and the practice. Although teachers are constantly encouraged by experts to implement the principles of dialogic teaching in the classroom, the academic concept of dialogic teaching has little impact on pedagogical practice (Mercer $\&$ Howe, 2002). There are many explanations, including the assumption that

$4 \quad$ Chapters 9 and 10 contain data material only. I understand that the authors leave space in these chapters for the readers to provide their own interpretations, but I see it as an impoverishment. As a reader, I am more curious about the interpretations offered by Lefstein and Snell.

5 Questions for readers are not a mere formal embellishment. The website provides an access key for the book to readers who can discuss possible interpretations of the events with the authors. 
it is difficult for teachers to teach in a dialogic way because they themselves were educated in the traditional monologic way. The reviewed book is thus a sort of a payback of the debt from academic authors to teachers. There is obvious potential for its use in teacher education, and in the final chapter, aptly titled "Do It Yourself", Lefstein and Snell encourage the teacher readers to set up self-help education groups and instruct them how to work in these groups with the book and, more generally, with the video recordings of teaching in order to improve their own practices. An alternative to education by best practice is the mutual sharing of data and experiences among teachers. Although their own teaching may be imperfect, ${ }^{6}$ reflecting on it may lead to the development of sensitivity, interpretation, and judgment, which are fundamental to the productive use of dialogic teaching methods. ${ }^{7}$

Although the publication is primarily written for practicing teachers, even academic experts dedicated to dialogic pedagogy, classroom discourse, teaching methods, and teachers' professional development will find it useful. The theoretical introduction (Chapters 1 and 2) is rather sparing and the analysis itself is unequivocally data driven. The authors possess theoretical erudition, without which they would be unable to conduct the analysis at this level. ${ }^{8}$ The quotations from the literature are mostly placed in footnotes, and the book can be read and understood even without referring to them. It is a very unpretentious way of presenting research, which does not diminish the value of the knowledge and ideas in the text. Simply put: the book is easy to read but encourages deep reflection on the phenomena it presents. The multi-voiced and multilayered way in which the book is structured leads the readers to ask numerous questions and provide their own answers which are immediately made relative leading to new questions and a search for more accurate answers. The book is concerned with many aspects of dialogic teaching, yet themes can be identified that occur throughout the whole publication and recur in different variations. It seems to me that student participation is the dominant theme. This is probably in accordance with the intentions of the authors, who state (page 135): "Dialogic pedagogy rests on

\footnotetext{
6 Lefstein and Snell argue that it is necessary to work with real-life material and come to terms with the fact that when implementing a detailed analysis, the process is not perfect.

7 Some indications of the best practice approach appear in the book; see e.g. the rules for organizing classroom debates on pages 129-130.

8 Against the background of the presented dilemmas, the question often arises of how to simultaneously fulfill various idealized features of dialogic teaching as they are defined in the theory by Alexander (2006), according to whom dialogic teaching is to be at the same time: collective, reciprocal, supportive, cumulative, and purposeful.
} 
the assumption that children learn best through participation in rich and challenging classroom discourse, and therefore requires that all students be encouraged to participate in such activity." In dialogic teaching, the teacher recedes into the background and to a certain extent "cleans out" the communication space, enabling the students to fill it. Lefstein and Snell show repeatedly (the topic is raised in Chapters 5, 6, 7, and 8) that the actual opening of the communication space does not provide for equal participation of the students. Some students are able to use the opportunities the teacher creates for them better than others; coincidentally, they are the students who would be able to take the floor even in a traditionally conceived lesson. The inclusiveness of the classroom dialogue affects both the shy and unassertive students and the students with lower levels of ability. The book does not give a clear answer to the question of how to involve all students in a sufficiently respectful manner and at the same time support their development.

This is, however, in accordance with the concept of the whole book, which conceives dialogic teaching as an issue to be discussed with the support of real-life data from the school environment. The book is excellently positioned to stimulate discussions of dialogic teaching.

\section{References}

Alexander, R. (2006). Towards dialogic teaching: rethinking classroom talk. Cambridge: Dialogos.

Mehan, H. (1979). Learning lessons: Social organisation in the classroom. Cambridge: Harvard University Press.

Mercer, N., \& Howe, C. (2012). Explaining the dialogic processes of teaching and learning: The value and potential of sociocultural theory. Learning, Culture and Social Interaction, 1(1) 12-21.

\section{Corresponding author}

\section{Klára Šed'ová}

Department of Educational Sciences, Faculty of Arts, Masaryk University

E-mail:ksedova@phil.muni.cz 
